\title{
Predominance of influenza A(H1N1)pdm09 virus genetic subclade 6B.1 and influenza B/Victoria lineage viruses at the start of the 2015/16 influenza season in Europe
}

\author{
E Broberg ${ }^{1}$, A Melidou ${ }^{2}$, K Prosenc ${ }^{3}$, K Bragstad ${ }^{4}, 0$ Hungnes ${ }^{4}$, on behalf of the WHO European Region and the European \\ Influenza Surveillance Network members of the reporting countries ${ }^{5}$ \\ 1. European Centre for Disease Prevention and Control (ECDC), Stockholm, Sweden \\ 2. National Influenza Centre for northern Greece, Microbiology Department, School of Medicine, Aristotle University of \\ Thessaloniki, Greece \\ 3. Laboratory for Public Health Virology, National Laboratory for Health, Environment and Food, Slovenia \\ 4. Norwegian Institute of Public health, Department of Virology, Oslo, Norway \\ 5. The members of the network who provided data are listed at the end of the article
}

Correspondence: Eeva Broberg (eeva.broberg@ecdc.europa.eu)

Broberg E, Melidou A, Prosenc K, Bragstad K, Hungnes O, on behalf of the WHO European Region and the European Influenza Surveillance Network members of the reporting countries. Predominance of influenza $\mathrm{A}\left(\mathrm{H}_{1} \mathrm{~N}_{1}\right)$ pdmog virus genetic subclade $6 \mathrm{~B} .1$ and influenza $\mathrm{B} /$ Victoria lineage viruses at the start of the $2015 / 16$ influenza season in Europe. Euro Surveill. 2016;21(13):pii=30184. DOI: http://dx.doi.org/10.2807/1560-7917.ES.2016.21.13.30184

Article submitted on og March 2016 / accepted on 31 March 2016 / published on 31 March 2016

Influenza $\mathrm{A}\left(\mathrm{H}_{1} \mathrm{~N}_{1}\right)$ pdmog viruses predominated in the European influenza 2015/16 season. Most analysed viruses clustered in a new genetic subclade 6B.1, antigenically similar to the northern hemisphere vaccine component $A / C a l i f o r n i a / 7 / 2009$. The predominant influenza B lineage was Victoria compared with Yamagata in the previous season. It remains to be evaluated at the end of the season if these changes affected the effectiveness of the vaccine for the 2015/16 season.

For the current northern hemisphere season, several reports have indicated intense influenza activity [1-5]. We analysed virological surveillance data from 20 European countries to study the genetic and antigenic characteristics of the circulating influenza viruses and compare them with the vaccine viruses and previously circulating strains.

\section{Virological influenza surveillance in Europe, influenza season 2015/16}

Virological influenza surveillance data in the World Health Organization (WHO) European Region are collected on a weekly basis and reported to The European Surveillance System (TESSy), a database hosted by the European Centre for Disease Prevention and Control (ECDC), as previously described [6]. From week 40/2015 to week 4/2016, 49 Member States of the Region reported influenza virus detections to TESSy, including 20 Member States (Belgium, Croatia, Czech Republic, Denmark, Finland, Germany, Greece, Ireland, Latvia, Netherlands, Norway, Portugal, Romania, Russia, Slovakia, Slovenia, Spain, Sweden, Switzerland, and the United Kingdom (UK)) that also reported antigenic or genetic characterisation data.

The antigenic and genetic reporting categories for TESSy are predefined by the WHO Collaborating Centre for Reference and Research on Influenza, London, for each influenza season. For antigenic characterisation, to denote a virus isolate as being like a vaccine or reference virus its haemagglutination inhibition ( $\mathrm{HI}$ ) titre with post-infection ferret antiserum raised against the reference virus should differ by no more than fourfold. For genetic characterisation, the allocation to reporting category is based on the phylogenetic and amino acid sequence analyses of haemagglutinin (HA) gene.

The summary analysis of the data are presented weekly in the Joint ECDC-WHO Regional Office for Europe weekly 'Flu News Europe' (http://flunewseurope.org/). Data on detections, antigenic and genetic characterisations were extracted on 8 February 2016 for analysis.

Between week 40/2015 and week 4/2016, influenza viruses were detected in 1,879 (19\%) of 9,882 sentinel specimens tested in the 20 countries also reporting on virus characterisation. Of these 1,879 specimens, $1,512(80 \%)$ were positive for type $A$ influenza virus and 367 (20\%) for type B. Of 1,441 subtyped influenza A viruses, 1,268 (88\%) were $A\left(\mathrm{H}_{1} \mathrm{~N}_{1}\right)$ pdmog. Of 129 type $B$ viruses with known lineage, 115 (89\%) were of the $B /$ Victoria/2/1987 lineage. 


\section{FIGURE 1}

Phylogenetic analysis of A(H1N1)pdm09 haemagglutinin (HA) nt sequences reported from European countries, between week 40/2015 and 4/2016

\section{Vaccine virus}

\section{Reference viruses}

Reported to TESSy as

A/South Africa/3626/2013 -

clade 6B

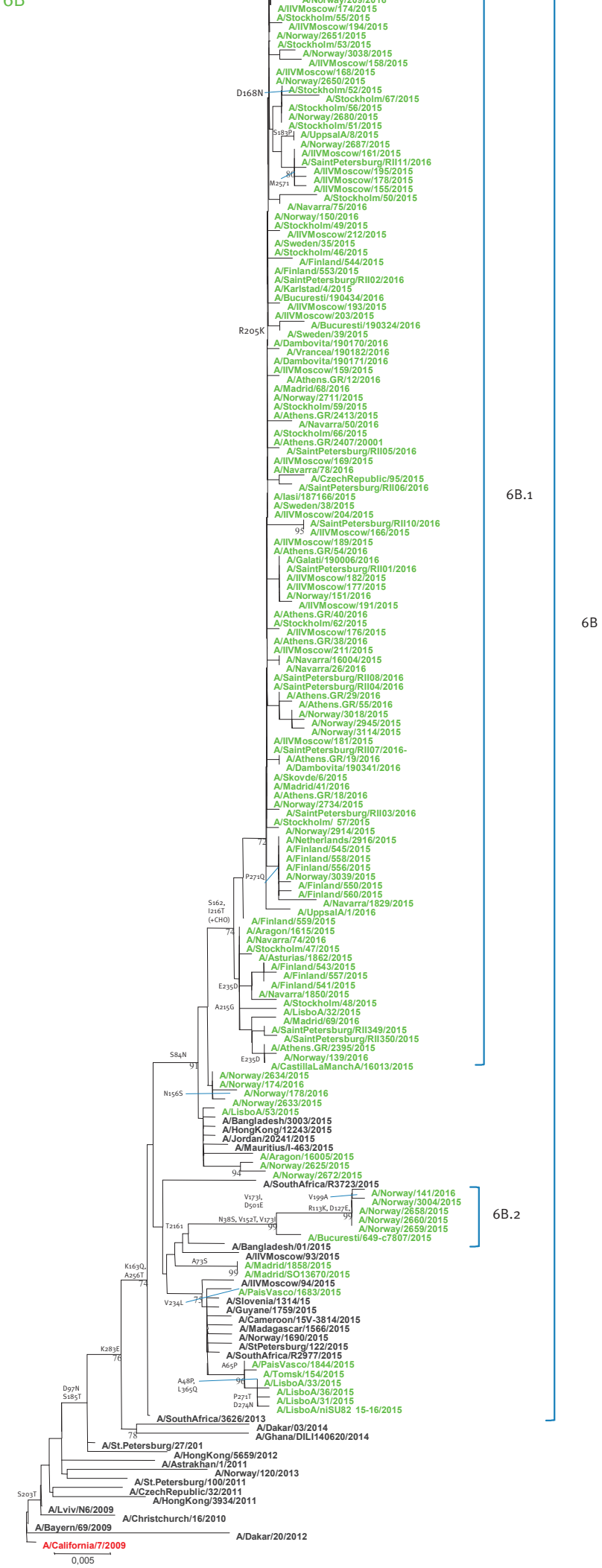

Some sequences obtained in this study were not used to construct the phylogenetic tree because they were identical and redundant. The sequences used for

the phylogenetic analysis were moreover only those of suitable length, and encode HA 1 amino acids $3-327$. These included sequences reported by the Czech Republic, Finland, Greece, Netherlands, Norway, Portugal, Romania, Russia, Slovenia, Spain and Sweden as well as sequences from reference A(H1N1)pdmo9 viruses. The tree was constructed with the neighbour-joining method, using Kimura-2 parameter-corrected distances and bootstrapped with 1,00o replicates, Molecular Evolutionary Genetics Analysis (MEGA) software version 5.0. 


\section{FIGURE 2}

Protein structure model (FluSurver-JSmol) of the haemagglutinin protein monomer of $\mathrm{A}(\mathrm{H} 1 \mathrm{~N} 1) \mathrm{pdm} 09$ subclade 6B.1, represented by A/Norway/2650/2015 (left), and subclade 6B.2, represented by A/Norway/2658/2015 (right)

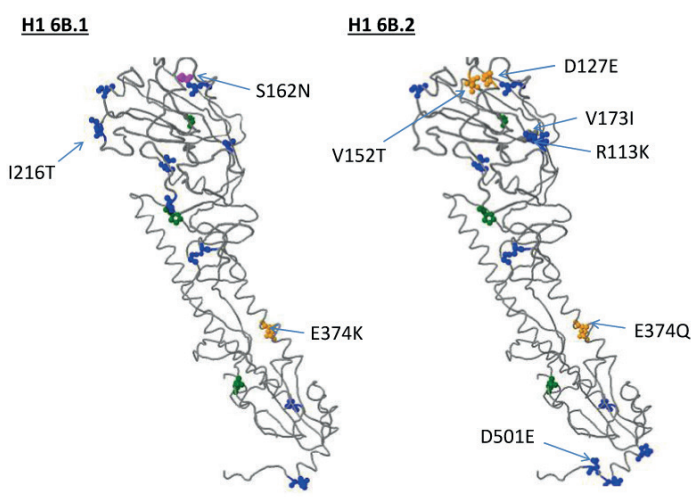

Amino acid differences compared with A/California/07/2009 are indicated in colour. Well-known differences are marked in blue. Common variant marker positions are indicated in green. Amino acid involved in virulence or antigenic drift is marked in orange. Amino acid not previously associated with a specific feature is marked in grey. Amino acid that creates a new potential $\mathrm{N}$-glycosylation site is marked in magenta.

\section{Virus characterisation}

Between weeks 40/2015 and 4/2016, 447 (24\%) of 1,879 influenza viruses were attributed to a genetic group by 16 countries (Belgium, Czech Republic, Denmark, Finland, Germany, Greece, Ireland, Netherlands, Norway, Portugal, Romania, Russia, Slovenia, Spain, Sweden and UK), and 429 (23\%) were attributed to an antigenic category by also 16 reporting countries (Croatia, Czech Republic, Denmark, Finland, Germany, Greece, Latvia, Netherlands, Portugal, Romania, Russia, Slovakia, Slovenia, Spain, Switzerland and UK) (Table 1).

The majority (68\%) of all genetic characterisations were reported from Norway $(n=84)$, Spain $(n=66)$, Germany $(n=54)$, Russia $(n=54)$ and Sweden $(n=46)$. The majority (70\%) of antigenic reports were from Russia $(n=124)$, Portugal $(n=99)$ and Germany $(n=78)$. For 150 viruses, reported in strain-based manner, both genetic and antigenic data were available.

All $313 \mathrm{~A}\left(\mathrm{H}_{1} \mathrm{~N}_{1}\right)$ pdmog viruses characterised genetically fell in clade 6 , subgroup $6 \mathrm{~B}$, represented by $A /$ South Africa/3626/2013. Viruses falling in this genetic subgroup, were all attributed to an antigenic category $\mathrm{A} /$ California/7/2009 that corresponds to the component included in the 2015/16 northern hemisphere vaccines.
Of the $77 \mathrm{~A}\left(\mathrm{H}_{3} \mathrm{~N}_{2}\right)$ viruses attributed to a genetic group, 50 (65\%) fell into genetic subgroup $3 \mathrm{C} .2 \mathrm{a}$ (represented by $\mathrm{A} /$ Hong Kong/4801/2014) that has been shown to be antigenically similar to $\mathrm{A} / \mathrm{Hong}$ Kong/4801/2014 and also to the current $A\left(\mathrm{H}_{3} \mathrm{~N}_{2}\right)$ vaccine virus A/Switzerland/9715293/2013 (Table 1). Twenty-six $A\left(\mathrm{H}_{3} \mathrm{~N}_{2}\right)$ viruses fell into the vaccine virus category of 3 C.3a subgroup. Viruses in subgroup 3 C.3 $\mathrm{b}$ (represented by A/Stockholm/28/2014) constituted a substantial part $(98 / 401)$ of the $\mathrm{A}\left(\mathrm{H}_{3} \mathrm{~N}_{2}\right)$ viruses in Europe in the $2014 / 15$ season [7], but none were yet reported by week 4/2016 (Table 1). Of $20 \mathrm{~A}\left(\mathrm{H}_{3} \mathrm{~N}_{2}\right)$ viruses attributed to an antigenic category, 14 were A/Switzerland/9715293/2013-like and thus similar to the northern hemisphere 2015/16 vaccine component and six were A/Hong Kong/4801/2014-like, similar to the southern hemisphere 2016 vaccine component and recommendation for northern hemisphere 2016/17 season.

All of the $44 \mathrm{~B} /$ Victoria lineage viruses characterised genetically to date fell in the clade $1 \mathrm{~A}$, represented by $\mathrm{B} /$ Brisbane/60/2008 which is included in quadrivalent vaccines for northern hemisphere $2015 / 16$. The $13 \mathrm{~B} /$ Yamagata lineage viruses all genetically resembled B/Phuket/3073/2013 recommended for inclusion in trivalent vaccines for northern hemisphere 2015/16. Thirty influenza $B$ viruses were antigenically characterised, 29 as B/Brisbane/60/2008-like and one as B/ Phuket/3073/2013-like.

Analysis of $\mathrm{A}\left(\mathrm{H}_{1} \mathrm{~N}_{1}\right)$ pdmog $\mathrm{HA}$ gene sequences from 12 countries (Czech Republic, Finland, Greece, Ireland, Netherlands, Norway, Portugal, Romania, Russia, Slovenia, Spain and Sweden) reported to TESSy, with provision of accession numbers in publicly accessible databases, confirmed that all these analysed viruses possessed the signature amino acid variations that define subgroup 6B viruses: D97N, K163Q, S185T, K283E and A256T [7-9]. All 215 analysed sequences, apart from two viruses isolated in Russia, also carried P83S and I321V substitutions in HA1.

The majority of sequences (173 of 215 TESSy-reported viruses) also possessed the amino acid signature of subclade 6B.1 and formed a separate branch in the phylogenetic analysis (Figure 1, Figure 2). The 6B.1 subclade is characterised by the amino acid substitutions S84N (present in a wider subgroup), S162N and I216T [8]. Six viruses carried amino acid substitutions V152T, V173l, D501E (the latter in $\mathrm{HA}_{2}$ ) characterising 6B.2 subclade. In addition the five most recently sampled of these six 6B.2 viruses all possessed the R113K, D127E and E374Q substitutions (Figure 1).

The highest number of accumulated variations in the known antigenic sites were observed in the antigenic site Ca. All subgroup 6B viruses possessed the $\mathrm{K}_{16} 6 \mathrm{Q}$ substitution, while the vast majority $(173 / 215 ; 80 \%)$ also possessed the $\mathrm{S}_{1} 6_{2} \mathrm{~N}$ substitution in $\mathrm{HA}_{1}$, resulting in a gain of a potential glycosylation site. Additional 
Viruses attributed to genetic and antigenic groups ${ }^{\text {a }}$, weeks 40/2015-04/2016

\begin{tabular}{|c|c|c|c|}
\hline Genetic group & $\begin{array}{l}\text { Number of } \\
\text { viruses }\end{array}$ & Antigenic group & $\begin{array}{l}\text { Number of } \\
\text { viruses }\end{array}$ \\
\hline $\mathrm{A}\left(\mathrm{H}_{1} \mathrm{~N}_{1}\right) \mathrm{pdmog} \mathrm{A} /$ South Africa/3626/2013 (subgroup 6B) ${ }^{\mathrm{b}}$ & 313 & $\mathrm{~A}\left(\mathrm{H}_{1} \mathrm{N1}\right) \mathrm{pdmog} \mathrm{A} / \mathrm{California/7/2009-like}$ & 379 \\
\hline $\mathrm{A}\left(\mathrm{H}_{3} \mathrm{~N}_{2}\right) \mathrm{A} /$ Hong Kong/4801/2014 (subgroup 3C.2a) ${ }^{\mathrm{b}}$ & 50 & $\mathrm{~A}\left(\mathrm{H}_{3} \mathrm{~N}_{2}\right) \mathrm{A} /$ Hong Kong/4801/2014-like & 6 \\
\hline $\mathrm{A}\left(\mathrm{H}_{3} \mathrm{~N}_{2}\right) \mathrm{A} / \mathrm{Samara} / 73 / 2013$ (subgroup $\left.{ }_{3} \mathrm{C} .3\right)^{\mathrm{c}}$ & 1 & $\begin{array}{c}\text { No separate antigenic category; expected to } \\
\text { resemble A/Stockholm/28/2014 }\end{array}$ & - \\
\hline $\mathrm{A}\left(\mathrm{H}_{3} \mathrm{~N}_{2}\right) \mathrm{A} /$ Stockholm/28/2014 (subgroup $\left.3 \mathrm{C} .3 \mathrm{~b}\right)^{\mathrm{c}}$ & o & $\mathrm{A}\left(\mathrm{H}_{3} \mathrm{~N}_{2}\right) \mathrm{A} / \mathrm{Stockholm} / 28 / 2014$-like & o \\
\hline $\mathrm{A}\left(\mathrm{H}_{3} \mathrm{~N}_{2}\right) \mathrm{A} /$ Switzerland/9715293/2013 (subgroup 3C.3a) ${ }^{\mathrm{b}}$ & 26 & $\mathrm{~A}\left(\mathrm{H}_{3} \mathrm{~N}_{2}\right) \mathrm{A} / \mathrm{S}$ witzerland/9715293/2013 & 14 \\
\hline B/Phuket/3073/2013 (Yamagata lineage clade 3$)^{\mathrm{b}}$ & 13 & B/Phuket/3073/2013 (Yamagata lineage) -like & 1 \\
\hline $\mathrm{B} /$ Brisbane/60/2008 $(\text { Victoria lineage clade } 1 \mathrm{~A})^{\mathrm{d}}$ & 44 & B/Brisbane/60/2008 (Victoria lineage) -like & 29 \\
\hline
\end{tabular}

The viruses which were genetically characterised are not necessarily the same than the viruses that were antigenically characterised.

${ }^{a}$ Genetic and antigenic groups used for reporting into The European Surveillance System are defined by World Health Organization Collaborating Centre for Reference and Research on Influenza for each influenza season. For antigenic characterisation, to denote a virus isolate as being like a vaccine or reference virus its haemagglutination inhibition (HI) titre with post-infection ferret antiserum raised against the reference virus should differ by no more than fourfold. For genetic characterisation, the allocation to reporting category is based on the phylogenetic and amino acid sequence analyses of haemagglutinin (HA) gene.

${ }^{b}$ These genetic groups contain viruses with antigenic properties similar to the viruses included in the trivalent influenza vaccine for $2015 / 16$.

' These genetic groups contain viruses with antigenic properties dissimilar to the viruses included in the trivalent influenza vaccine for 2015/16.

${ }^{d}$ Viruses in this genetic group have antigenic properties similar to those of the vaccine component (B/Brisbane/60/2008) recommended for use in quadrivalent influenza vaccines for 2015/16.

variations observed were S162K, D168N, K170E, R205K, A215G, E235D and a partial A139D. Cb antigenic site variation $\mathrm{A} 73 \mathrm{~S}$ was observed in four viruses from Spain, one of which also possessed substitution N156K in Sa antigenic site. Another Norwegian virus had a N156S substitution in Sa antigenic site. Notably, all 6B.2 viruses and also two of the 6B viruses not belong. ing to any of the newly identified subgroups possessed substitutions affecting the loop that consists of amino acid positions 151 to 159 located adjacent to the receptor binding site.

When comparing the $A\left(\mathrm{H}_{1} \mathrm{~N}_{1}\right)$ pdmog strains with the corresponding strain in the current northern hemisphere influenza vaccine, $A / C a l i f o r n i a / 7 / 2009$, the $H A 1$ sequences (nt 1-981, amino acids 1-327) exhibited nt similarity of 96.8 to $98.0 \%$ and deduced amino acid similarity of 95.4 to $96.3 \%$. Viruses within subclade 6B.1 exhibited higher HA nt heterogeneity, with similarities ranging between 98.8 and $100 \%$, while within subclade 6B.2 strains exhibited higher nt similarity, ranging between 99.3 and $100 \%$, as the group consists of fewer sequences and most of them from one region only. The viruses analysed phylogenetically are listed in Table 2.

\section{Discussion}

Continuous surveillance of influenza viruses is essential for detecting emerging new variant strains and providing viruses for vaccine production [10]. In Europe, within the detected $A$ subtypes, influenza $A\left(\mathrm{H}_{1} \mathrm{~N}_{1}\right)$ pdmog predominated during 2010/11, 2012/13 and 2013/14 seasons and concerned $97 \%$ [11], 62\% [12] and $53 \%$ [13] of subtyped influenza viruses respectively, with variation in country-specific proportions. The
$\mathrm{A}\left(\mathrm{H}_{1} \mathrm{~N}_{1}\right)$ pdmog vaccine component $\mathrm{A} / \mathrm{California/7/2009}$ has not been changed since the 2009 pandemic and the circulating $A\left(\mathrm{H}_{1} \mathrm{~N}_{1}\right)$ pdmog viruses have remained antigenically similar to the virus included in the vaccines throughout the influenza 2009/10 to 2015/16 seasons. However, since 2013, several reports have indicated the emergence of an expanding subgroup of $\mathrm{A}\left(\mathrm{H}_{1} \mathrm{~N}_{1}\right)$ pdmog viruses, designated $6 \mathrm{~B}[1,8,9]$. This subgroup appeared in 2012/13 and became predominant in 2013/14 [14].

In this study, we observe the further emergence of a subclade within the 6B subgroup, designated 6B.1 [15], which accounted for the majority of the $\mathrm{A}\left(\mathrm{H}_{1} \mathrm{~N}_{1}\right)$ pdmog viruses detected across the WHO European Region during the first weeks of the 2015/16 influenza season. In addition, the surveillance data show a change in the predominant $B$ virus lineage from B/Yamagata which predominated in the preceding three seasons in Europe to B/Victoria.

Our data are preliminary for this season and are based on influenza surveillance without detailed reporting of clinical symptoms or vaccination status. Our genetic analysis was only based on the HA gene and does not extend to changes e.g. in genes encoding internal proteins of influenza viruses. The data reported to TESSy do not include antigenic titres and therefore no direct analysis of antigenic properties was possible. However, the antigenic reports rely on national influenza centres' antigenic analysis that the viruses reported as like to vaccine virus were not more than fourfold different in $\mathrm{HI}$ titres from the vaccine or reference viruses. 
TABLE 2A

Details of the A(H1N1)pdm09 sequences retrieved from the Global Initiative on Sharing All Influenza Data (GISAID)'s EpiFlu Database or GenBank, for haemagglutinin-gene-based phylogenetic analysis in this study

\begin{tabular}{|c|c|c|c|c|c|}
\hline ID & Country & Strain name & $\begin{array}{l}\text { Collection } \\
\text { date }\end{array}$ & Originating laboratory & Submitting laboratory \\
\hline EPI685415 & Netherlands & A/Netherlands/2916/2015 & $6 / 11 / 2015$ & $\begin{array}{l}\text { National Institute for Public } \\
\text { Health and the Environment } \\
\text { (RIVM) }\end{array}$ & $\begin{array}{l}\text { National Institute for Public } \\
\text { Health and the Environment } \\
\text { (RIVM) }\end{array}$ \\
\hline EPI674853 & Sweden & A/Stockholm/46/2015 & $9 / 10 / 2015$ & - & $\begin{array}{c}\text { Swedish Institute for Infectious } \\
\text { Disease Control }\end{array}$ \\
\hline EPI674745 & Sweden & A/Stockholm/47/2015 & $22 / 10 / 2015$ & - & $\begin{array}{c}\text { Swedish Institute for Infectious } \\
\text { Disease Control }\end{array}$ \\
\hline EPI674753 & Sweden & A/Stockholm/48/2015 & $24 / 10 / 2015$ & - & $\begin{array}{c}\text { Swedish Institute for Infectious } \\
\text { Disease Control }\end{array}$ \\
\hline EPI674841 & Sweden & A/Karlstad/4/2015 & $25 / 10 / 2015$ & - & $\begin{array}{c}\text { Swedish Institute for Infectious } \\
\text { Disease Control }\end{array}$ \\
\hline EPI674777 & Sweden & A/Stockholm/49/2015 & $7 / 11 / 2015$ & - & $\begin{array}{c}\text { Swedish Institute for Infectious } \\
\text { Disease Control }\end{array}$ \\
\hline EPI686820 & Sweden & A/Skovde/6/2015 & $18 / 11 / 2015$ & - & $\begin{array}{c}\text { Swedish Institute for Infectious } \\
\text { Disease Control }\end{array}$ \\
\hline EPI686772 & Sweden & A/Stockholm/57/2015 & $18 / 11 / 2015$ & - & $\begin{array}{c}\text { Swedish Institute for Infectious } \\
\text { Disease Control }\end{array}$ \\
\hline EPI674785 & Sweden & A/Stockholm/50/2015 & $11 / 11 / 2015$ & - & $\begin{array}{c}\text { Swedish Institute for Infectious } \\
\text { Disease Control }\end{array}$ \\
\hline EPI674793 & Sweden & A/Stockholm/51/2015 & $11 / 11 / 2015$ & - & $\begin{array}{c}\text { Swedish Institute for Infectious } \\
\text { Disease Control }\end{array}$ \\
\hline EPI674801 & Sweden & A/Stockholm/52/2015 & $11 / 11 / 2015$ & - & $\begin{array}{c}\text { Swedish Institute for Infectious } \\
\text { Disease Control }\end{array}$ \\
\hline EPI674847 & Sweden & A/Stockholm/53/2015 & $12 / 11 / 2015$ & - & $\begin{array}{c}\text { Swedish Institute for Infectious } \\
\text { Disease Control }\end{array}$ \\
\hline EPI674809 & Sweden & A/Stockholm/55/2015 & $10 / 11 / 2015$ & - & $\begin{array}{c}\text { Swedish Institute for Infectious } \\
\text { Disease Control }\end{array}$ \\
\hline EPI686764 & Sweden & A/Stockholm/56/2015 & $18 / 11 / 2015$ & - & $\begin{array}{c}\text { Swedish Institute for Infectious } \\
\text { Disease Control }\end{array}$ \\
\hline EPI686799 & Sweden & A/Stockholm/59/2015 & $19 / 11 / 2015$ & - & $\begin{array}{c}\text { Swedish Institute for Infectious } \\
\text { Disease Control }\end{array}$ \\
\hline EPI686828 & Sweden & A/Stockholm/6o/2015 & $25 / 11 / 2015$ & - & $\begin{array}{c}\text { Swedish Institute for Infectious } \\
\text { Disease Control }\end{array}$ \\
\hline EPI686844 & Sweden & A/Stockholm/62/2015 & $26 / 11 / 2015$ & - & $\begin{array}{c}\text { Swedish Institute for Infectious } \\
\text { Disease Control }\end{array}$ \\
\hline EPI687173 & Sweden & A/Stockholm/66/2015 & $23 / 11 / 2015$ & - & $\begin{array}{c}\text { Swedish Institute for Infectious } \\
\text { Disease Control }\end{array}$ \\
\hline EPI687199 & Sweden & A/Stockholm/67/2015 & $21 / 11 / 2015$ & - & $\begin{array}{c}\text { Swedish Institute for Infectious } \\
\text { Disease Control }\end{array}$ \\
\hline EPI674825 & Sweden & A/Sweden/35/2015 & $12 / 11 / 2015$ & - & $\begin{array}{c}\text { Swedish Institute for Infectious } \\
\text { Disease Control }\end{array}$ \\
\hline EPI686852 & Sweden & A/Uppsala/8/2015 & $27 / 11 / 2015$ & - & $\begin{array}{c}\text { Swedish Institute for Infectious } \\
\text { Disease Control }\end{array}$ \\
\hline EPI686892 & Sweden & A/Sweden/37/2015 & $26 / 11 / 2015$ & - & $\begin{array}{c}\text { Swedish Institute for Infectious } \\
\text { Disease Control }\end{array}$ \\
\hline EPI686900 & Sweden & A/Sweden/38/2015 & $2 / 12 / 2015$ & - & $\begin{array}{c}\text { Swedish Institute for Infectious } \\
\text { Disease Control }\end{array}$ \\
\hline EPI686908 & Sweden & A/Sweden/39/2015 & $2 / 12 / 2015$ & - & $\begin{array}{c}\text { Swedish Institute for Infectious } \\
\text { Disease Control }\end{array}$ \\
\hline EPI694343 & Sweden & A/Uppsala/1/2016 & $11 / 1 / 2016$ & - & $\begin{array}{c}\text { Swedish Institute for Infectious } \\
\text { Disease Control }\end{array}$ \\
\hline EPI671518 & Norway & A/Norway/2625/2015 & $21 / 10 / 2015$ & $\begin{array}{c}\text { Sorlandet Sykehus HF, Dept. of } \\
\text { Medical Microbiology }\end{array}$ & $\begin{array}{c}\text { Norwegian Institute of Public } \\
\text { Health }\end{array}$ \\
\hline EPI675750 & Norway & A/Norway/2659/2015 & $3 / 11 / 2015$ & $\begin{array}{l}\text { Drammen Hospital / Vestreviken } \\
\text { HF, Department for Medical } \\
\text { Microbiology section Drammen }\end{array}$ & $\begin{array}{c}\text { Norwegian Institute of Public } \\
\text { Health }\end{array}$ \\
\hline EPI675751 & Norway & A/Norway/266o/2015 & $3 / 11 / 2015$ & $\begin{array}{l}\text { Drammen Hospital / Vestreviken } \\
\text { HF, Department for Medical } \\
\text { Microbiology section Drammen }\end{array}$ & $\begin{array}{l}\text { Norwegian Institute of Public } \\
\text { Health }\end{array}$ \\
\hline
\end{tabular}

ID: identity; SAR: Special Administrative Region; WHO: World Health Organization. 
TABLE 2B

Details of the A(H1N1)pdm09 sequences retrieved from the Global Initiative on Sharing All Influenza Data (GISAID)'s EpiFlu Database or GenBank, for haemagglutinin-gene-based phylogenetic analysis in this study

\begin{tabular}{|c|c|c|c|c|c|}
\hline ID & Country & Strain name & $\begin{array}{l}\text { Collection } \\
\text { date }\end{array}$ & Originating laboratory & Submitting laboratory \\
\hline EPI675754 & Norway & A/Norway/2672/2015 & $1 / 11 / 2015$ & $\begin{array}{l}\text { Oslo University Hospital, Ulleval } \\
\text { Hospital, Dept. of Microbiology }\end{array}$ & $\begin{array}{l}\text { Norwegian Institute of Public } \\
\text { Health }\end{array}$ \\
\hline EPI675756 & Norway & A/Norway/2680/2015 & $12 / 11 / 2015$ & $\begin{array}{c}\text { Ostfold Hospital - Fredrikstad, } \\
\text { Dept. of Microbiology }\end{array}$ & $\begin{array}{c}\text { Norwegian Institute of Public } \\
\text { Health }\end{array}$ \\
\hline EPI67576o & Norway & A/Norway/2687/2015 & $13 / 11 / 2015$ & - & $\begin{array}{c}\text { Norwegian Institute of Public } \\
\text { Health }\end{array}$ \\
\hline EPI695284 & Norway & A/Norway/2711/2015 & $18 / 11 / 2015$ & - & $\begin{array}{c}\text { Norwegian Institute of Public } \\
\text { Health }\end{array}$ \\
\hline EPI695299 & Norway & A/Norway/2914/2015 & $14 / 12 / 2015$ & $\begin{array}{c}\text { Sorlandet Sykehus HF, Dept. of } \\
\text { Medical Microbiology }\end{array}$ & $\begin{array}{c}\text { Norwegian Institute of Public } \\
\text { Health }\end{array}$ \\
\hline EPI695310 & Norway & A/Norway/3004/2015 & $15 / 12 / 2015$ & $\begin{array}{l}\text { Innlandet Hospital Trust, } \\
\text { Division Lillehammer, } \\
\text { Department for Microbiology }\end{array}$ & $\begin{array}{c}\text { Norwegian Institute of Public } \\
\text { Health }\end{array}$ \\
\hline EPI695311 & Norway & A/Norway/3018/2015 & $26 / 12 / 2015$ & - & $\begin{array}{l}\text { Norwegian Institute of Public } \\
\text { Health }\end{array}$ \\
\hline EPI695313 & Norway & A/Norway/3038/2015 & $26 / 12 / 2015$ & Aalesund sjukehus & $\begin{array}{l}\text { Norwegian Institute of Public } \\
\text { Health }\end{array}$ \\
\hline EPI695343 & Norway & A/Norway/174/2016 & $7 / 1 / 2016$ & $\begin{array}{l}\text { St. Olavs Hospital HF, Dept. of } \\
\text { Medical Microbiology }\end{array}$ & $\begin{array}{l}\text { Norwegian Institute of Public } \\
\text { Health }\end{array}$ \\
\hline EPI695344 & Norway & A/Norway/178/2016 & $6 / 1 / 2016$ & $\begin{array}{l}\text { Health Forde, Department of } \\
\text { Microbiology }\end{array}$ & $\begin{array}{c}\text { Norwegian Institute of Public } \\
\text { Health }\end{array}$ \\
\hline EPI695349 & Norway & A/Norway/209/2016 & $8 / 1 / 2016$ & $\begin{array}{l}\text { Stavanger Universitetssykehus, } \\
\text { Avd. for Medisinsk Mikrobiologi }\end{array}$ & $\begin{array}{c}\text { Norwegian Institute of Public } \\
\text { Health }\end{array}$ \\
\hline EPI677648 & Finland & A/Finland/541/2015 & $9 / 11 / 2015$ & $\begin{array}{c}\text { Helsinki University Central } \\
\text { Hospital, Laboratory Services } \\
\text { (HUSLAB) }\end{array}$ & $\begin{array}{c}\text { National Institute for Health and } \\
\text { Welfare }\end{array}$ \\
\hline EPI677651 & Finland & A/Finland/543/2015 & $19 / 11 / 2015$ & $\begin{array}{l}\text { Helsinki University Central } \\
\text { Hospital, Laboratory Services } \\
\text { (HUSLAB) }\end{array}$ & $\begin{array}{l}\text { National Institute for Health and } \\
\text { Welfare }\end{array}$ \\
\hline EPI678232 & Finland & A/Finland/544/2015 & $13 / 11 / 2015$ & $\begin{array}{c}\text { Helsinki University Central } \\
\text { Hospital, Laboratory Services } \\
\text { (HUSLAB) }\end{array}$ & $\begin{array}{c}\text { National Institute for Health and } \\
\text { Welfare }\end{array}$ \\
\hline EPI696158 & Russia & A/Tomsk/154/2015 & $19 / 11 / 2015$ & $\begin{array}{c}\text { D.I. Ivanovsky Research } \\
\text { Institute of virology MoPH of } \\
\text { RF,Moscow }\end{array}$ & $\begin{array}{c}\text { WHO National Influenza Centre } \\
\text { Russian Federation }\end{array}$ \\
\hline EPI696470 & Russia & A/IIV-Moscow/211/2015 & $23 / 12 / 2015$ & $\begin{array}{c}\text { D.I. Ivanovsky Research } \\
\text { Institute of virology MoPH of } \\
\text { RF,Moscow }\end{array}$ & $\begin{array}{l}\text { WHO National Influenza Centre } \\
\text { Russian Federation }\end{array}$ \\
\hline EPI696478 & Russia & A/IIV-Moscow/212/2015 & $23 / 12 / 2015$ & $\begin{array}{c}\text { D.I. Ivanovsky Research } \\
\text { Institute of virology MoPH of } \\
\text { RF,Moscow }\end{array}$ & $\begin{array}{c}\text { WHO National Influenza Centre } \\
\text { Russian Federation }\end{array}$ \\
\hline EPI690291 & Spain & A/Aragon/16005/2015 & $21 / 12 / 2015$ & $\begin{array}{c}\text { Servicio de Microbiología } \\
\text { Hospital Miguel Servet }\end{array}$ & Instituto de Salud Carlos III \\
\hline EPI671520 & Norway & A/Norway/2631/2015 & $26 / 10 / 2015$ & $\begin{array}{c}\text { Sorlandet Sykehus HF, Dept. of } \\
\text { Medical Microbiology }\end{array}$ & $\begin{array}{c}\text { Norwegian Institute of Public } \\
\text { Health }\end{array}$ \\
\hline EPI671521 & Norway & A/Norway/2633/2015 & $27 / 10 / 2015$ & $\begin{array}{l}\text { Haukeland University Hospital, } \\
\text { Dept. of Microbiology }\end{array}$ & $\begin{array}{c}\text { Norwegian Institute of Public } \\
\text { Health }\end{array}$ \\
\hline EPI671522 & Norway & A/Norway/2634/2015 & $27 / 10 / 2015$ & $\begin{array}{l}\text { Haukeland University Hospital, } \\
\text { Dept. of Microbiology }\end{array}$ & $\begin{array}{c}\text { Norwegian Institute of Public } \\
\text { Health }\end{array}$ \\
\hline EPI671525 & Norway & A/Norway/2650/2015 & $3 / 11 / 2015$ & $\begin{array}{c}\text { Ostfold Hospital - Fredrikstad, } \\
\text { Dept. of Microbiology }\end{array}$ & $\begin{array}{c}\text { Norwegian Institute of Public } \\
\text { Health }\end{array}$ \\
\hline EPI675748 & Norway & A/Norway/2651/2015 & $2 / 11 / 2015$ & $\begin{array}{l}\text { Mikrobiologisk laboratorium, } \\
\text { Sykehuset i Vestfold }\end{array}$ & $\begin{array}{c}\text { Norwegian Institute of Public } \\
\text { Health }\end{array}$ \\
\hline EPI675749 & Norway & A/Norway/2658/2015 & $4 / 11 / 2015$ & $\begin{array}{c}\text { Drammen Hospital / Vestreviken } \\
\text { HF, Department for Medical } \\
\text { Microbiology section Drammen } \\
\end{array}$ & $\begin{array}{c}\text { Norwegian Institute of Public } \\
\text { Health }\end{array}$ \\
\hline EPI695334 & Norway & A/Norway/139/2016 & $4 / 1 / 2016$ & $\begin{array}{c}\text { Haukeland University Hospital, } \\
\text { Dept. of Microbiology }\end{array}$ & $\begin{array}{c}\text { Norwegian Institute of Public } \\
\text { Health }\end{array}$ \\
\hline EPI695336 & Norway & A/Norway/141/2016 & $4 / 1 / 2016$ & $\begin{array}{c}\text { Unilabs Telelab, Laboratory for } \\
\text { Medical Microbiology }\end{array}$ & $\begin{array}{l}\text { Norwegian Institute of Public } \\
\text { Health }\end{array}$ \\
\hline
\end{tabular}

ID: identity; SAR: Special Administrative Region; WHO: World Health Organization. 
TABLE 2C

Details of the A(H1N1)pdm09 sequences retrieved from the Global Initiative on Sharing All Influenza Data (GISAID)'s EpiFlu Database or GenBank, for haemagglutinin-gene-based phylogenetic analysis in this study

\begin{tabular}{|c|c|c|c|c|c|}
\hline ID & Country & Strain name & $\begin{array}{l}\text { Collection } \\
\text { date }\end{array}$ & Originating laboratory & Submitting laboratory \\
\hline EPI695339 & Norway & A/Norway/150/2016 & $12 / 1 / 2016$ & $\begin{array}{l}\text { Stavanger Universitetssykehus, } \\
\text { Avd. for Medisinsk Mikrobiologi }\end{array}$ & $\begin{array}{c}\text { Norwegian Institute of Public } \\
\text { Health }\end{array}$ \\
\hline EPI695340 & Norway & A/Norway/151/2016 & $12 / 1 / 2016$ & $\begin{array}{l}\text { Stavanger Universitetssykehus, } \\
\text { Avd. for Medisinsk Mikrobiologi }\end{array}$ & $\begin{array}{c}\text { Norwegian Institute of Public } \\
\text { Health }\end{array}$ \\
\hline EPI695287 & Norway & A/Norway/2734/2015 & $13 / 11 / 2015$ & $\begin{array}{c}\text { Innlandet Hospital Trust, } \\
\text { Division Lillehammer, } \\
\text { Department for Microbiology }\end{array}$ & $\begin{array}{c}\text { Norwegian Institute of Public } \\
\text { Health }\end{array}$ \\
\hline EPI695304 & Norway & A/Norway/2945/2015 & $16 / 12 / 2015$ & - & $\begin{array}{c}\text { Norwegian Institute of Public } \\
\text { Health }\end{array}$ \\
\hline EPI695314 & Norway & A/Norway/3039/2015 & $28 / 12 / 2015$ & Aalesund sjukehus & $\begin{array}{c}\text { Norwegian Institute of Public } \\
\text { Health }\end{array}$ \\
\hline EPI695326 & Norway & A/Norway/3114/2015 & $28 / 12 / 2015$ & $\begin{array}{l}\text { Drammen Hospital / Vestreviken } \\
\text { HF, Department for Medical } \\
\text { Microbiology section Drammen }\end{array}$ & $\begin{array}{c}\text { Norwegian Institute of Public } \\
\text { Health }\end{array}$ \\
\hline EPI678234 & Finland & A/Finland/545/2015 & $19 / 11 / 2015$ & $\begin{array}{c}\text { Helsinki University Central } \\
\text { Hospital, Laboratory Services } \\
\text { (HUSLAB) }\end{array}$ & $\begin{array}{c}\text { National Institute for Health and } \\
\text { Welfare }\end{array}$ \\
\hline EPI678238 & Finland & A/Finland/550/2015 & $4 / 12 / 2015$ & $\begin{array}{l}\text { National Institute for Health and } \\
\text { Welfare }\end{array}$ & $\begin{array}{c}\text { National Institute for Health and } \\
\text { Welfare }\end{array}$ \\
\hline EPI678240 & Finland & A/Finland/553/2015 & $6 / 12 / 2015$ & NordLab Oulu & $\begin{array}{c}\text { National Institute for Health and } \\
\text { Welfare }\end{array}$ \\
\hline EPI693689 & Finland & A/Finland/556/2015 & $16 / 12 / 2015$ & $\begin{array}{l}\text { National Institute for Health and } \\
\text { Welfare }\end{array}$ & $\begin{array}{c}\text { National Institute for Health and } \\
\text { Welfare }\end{array}$ \\
\hline EPI687734 & Finland & A/Finland/557/2015 & $15 / 12 / 2015$ & $\begin{array}{c}\text { National Institute for Health and } \\
\text { Welfare }\end{array}$ & $\begin{array}{c}\text { National Institute for Health and } \\
\text { Welfare }\end{array}$ \\
\hline EPI693690 & Finland & A/Finland/558/2015 & $19 / 12 / 2015$ & $\begin{array}{l}\text { National Institute for Health and } \\
\text { Welfare }\end{array}$ & $\begin{array}{l}\text { National Institute for Health and } \\
\text { Welfare }\end{array}$ \\
\hline EPI693691 & Finland & A/Finland/559/2015 & $14 / 12 / 2015$ & $\begin{array}{c}\text { National Institute for Health and } \\
\text { Welfare }\end{array}$ & $\begin{array}{c}\text { National Institute for Health and } \\
\text { Welfare }\end{array}$ \\
\hline EPI693692 & Finland & A/Finland/56o/2015 & $18 / 12 / 2015$ & $\begin{array}{l}\text { National Institute for Health and } \\
\text { Welfare }\end{array}$ & $\begin{array}{c}\text { National Institute for Health and } \\
\text { Welfare }\end{array}$ \\
\hline EPI674284 & Portugal & A/Lisboa/31/2015 & $19 / 11 / 2015$ & Instituto Nacional de Saude & $\begin{array}{c}\text { INSA National Institute of Health } \\
\text { Portugal }\end{array}$ \\
\hline EPI674285 & Portugal & A/Lisboa/32/2015 & $18 / 11 / 2015$ & Instituto Nacional de Saude & $\begin{array}{c}\text { INSA National Institute of Health } \\
\text { Portugal }\end{array}$ \\
\hline EPI678690 & Portugal & A/Lisboa/33/2015 & $25 / 11 / 2015$ & Instituto Nacional de Saude & $\begin{array}{c}\text { INSA National Institute of Health } \\
\text { Portugal }\end{array}$ \\
\hline EPI678691 & Portugal & A/Lisboa/36/2015 & $2 / 12 / 2015$ & Instituto Nacional de Saude & $\begin{array}{c}\text { INSA National Institute of Health } \\
\text { Portugal }\end{array}$ \\
\hline EPI692997 & Portugal & A/Lisboa/53/2015 & $22 / 12 / 2015$ & Instituto Nacional de Saude & $\begin{array}{c}\text { INSA National Institute of Health } \\
\text { Portugal }\end{array}$ \\
\hline EPI678693 & Portugal & A/Lisboa/niSU82_15-16/2015 & $2 / 12 / 2015$ & Instituto Nacional de Saude & $\begin{array}{c}\text { INSA National Institute of Health } \\
\text { Portugal }\end{array}$ \\
\hline EPI699780 & Greece & A/Athens.GR/18/2016 & $4 / 1 / 2016$ & Hellenic Pasteur Institute & Hellenic Pasteur Institute \\
\hline EPI699778 & Greece & A/Athens.GR/19/2016 & $4 / 1 / 2016$ & Hellenic Pasteur Institute & Hellenic Pasteur Institute \\
\hline EPI699774 & Greece & A/Athens.GR/29/2016 & $7 / 1 / 2016$ & Hellenic Pasteur Institute & Hellenic Pasteur Institute \\
\hline EPI699772 & Greece & A/Athens.GR/38/2016 & $7 / 1 / 2016$ & Hellenic Pasteur Institute & Hellenic Pasteur Institute \\
\hline EPI699770 & Greece & A/Athens.GR/40/2016 & $7 / 1 / 2016$ & Hellenic Pasteur Institute & Hellenic Pasteur Institute \\
\hline EPI699766 & Greece & A/Athens.GR/54/2016 & $8 / 1 / 2016$ & Hellenic Pasteur Institute & Hellenic Pasteur Institute \\
\hline EPI699764 & Greece & A/Athens.GR/55/2016 & $8 / 1 / 2016$ & Hellenic Pasteur Institute & Hellenic Pasteur Institute \\
\hline EPI670326 & Romania & A/lasi/187166/2015 & $13 / 10 / 2015$ & Cantacuzino Institute & Cantacuzino Institute \\
\hline EPI690111 & Romania & A/Bucuresti/649-c7807/2015 & $22 / 12 / 2015$ & Cantacuzino Institute & Cantacuzino Institute \\
\hline EPI699023 & Romania & A/Bucuresti/190460/2016 & $19 / 1 / 2016$ & Cantacuzino Institute & Cantacuzino Institute \\
\hline EPI696174 & Russia & A/IIV-Moscow/158/2015 & $12 / 2015$ & $\begin{array}{c}\text { D.I. Ivanovsky Research } \\
\text { Institute of virology MoPH of } \\
\text { RF,Moscow }\end{array}$ & $\begin{array}{l}\text { WHO National Influenza Centre } \\
\text { Russian Federation }\end{array}$ \\
\hline
\end{tabular}


TABLE 2D

Details of the A(H1N1)pdm09 sequences retrieved from the Global Initiative on Sharing All Influenza Data (GISAID)'s EpiFlu Database or GenBank, for haemagglutinin-gene-based phylogenetic analysis in this study

\begin{tabular}{|c|c|c|c|c|c|}
\hline ID & Country & Strain name & $\begin{array}{l}\text { Collection } \\
\text { date }\end{array}$ & Originating laboratory & Submitting laboratory \\
\hline EPI696182 & Russia & A/IIV-Moscow/159/2015 & $12 / 2015$ & $\begin{array}{l}\text { D.I. Ivanovsky Research } \\
\text { Institute of virology MoPH of } \\
\text { RF,Moscow }\end{array}$ & $\begin{array}{l}\text { WHO National Influenza Centre } \\
\text { Russian Federation }\end{array}$ \\
\hline EPI696198 & Russia & A/IIV-Moscow/161/2015 & $14 / 12 / 2015$ & $\begin{array}{l}\text { D.I. Ivanovsky Research } \\
\text { Institute of virology MoPH of } \\
\text { RF,Moscow }\end{array}$ & $\begin{array}{l}\text { WHO National Influenza Centre } \\
\text { Russian Federation }\end{array}$ \\
\hline EPI696246 & Russia & A/IIV-Moscow/169/2015 & $17 / 12 / 2015$ & $\begin{array}{l}\text { D.I. Ivanovsky Research } \\
\text { Institute of virology MoPH of } \\
\text { RF,Moscow }\end{array}$ & $\begin{array}{l}\text { WHO National Influenza Centre } \\
\text { Russian Federation }\end{array}$ \\
\hline EPI696270 & Russia & A/IIV-Moscow/174/2015 & $16 / 12 / 2015$ & $\begin{array}{l}\text { D.I. Ivanovsky Research } \\
\text { Institute of virology MoPH of } \\
\text { RF,Moscow }\end{array}$ & $\begin{array}{l}\text { WHO National Influenza Centre } \\
\text { Russian Federation }\end{array}$ \\
\hline EPI696278 & Russia & A/IIV-Moscow/176/2015 & $15 / 12 / 2015$ & $\begin{array}{l}\text { D.I. Ivanovsky Research } \\
\text { Institute of virology MoPH of } \\
\text { RF,Moscow }\end{array}$ & $\begin{array}{l}\text { WHO National Influenza Centre } \\
\text { Russian Federation }\end{array}$ \\
\hline EPI696286 & Russia & A/IIV-Moscow/177/2015 & $16 / 12 / 2015$ & $\begin{array}{c}\text { D.I. Ivanovsky Research } \\
\text { Institute of virology MoPH of } \\
\text { RF,Moscow }\end{array}$ & $\begin{array}{l}\text { WHO National Influenza Centre } \\
\text { Russian Federation }\end{array}$ \\
\hline EPI696326 & Russia & A/IIV-Moscow/183/2015 & $21 / 12 / 2015$ & $\begin{array}{c}\text { D.I. Ivanovsky Research } \\
\text { Institute of virology MoPH of } \\
\text { RF,Moscow }\end{array}$ & $\begin{array}{l}\text { WHO National Influenza Centre } \\
\text { Russian Federation }\end{array}$ \\
\hline EPI696382 & Russia & A/IIV-Moscow/191/2015 & $20 / 12 / 2015$ & $\begin{array}{c}\text { D.I. Ivanovsky Research } \\
\text { Institute of virology MoPH of } \\
\text { RF,Moscow }\end{array}$ & $\begin{array}{l}\text { WHO National Influenza Centre } \\
\text { Russian Federation }\end{array}$ \\
\hline EPI696414 & Russia & A/IIV-Moscow/195/2015 & $22 / 12 / 2015$ & $\begin{array}{c}\text { D.I. Ivanovsky Research } \\
\text { Institute of virology MoPH of } \\
\text { RF,Moscow } \\
\end{array}$ & $\begin{array}{l}\text { WHO National Influenza Centre } \\
\text { Russian Federation }\end{array}$ \\
\hline EPI687093 & Russia & A/Saint-Petersburg/RII349/2015 & $25 / 11 / 2015$ & & $\begin{array}{c}\text { WHO National Influenza Centre } \\
\text { Russian Federation }\end{array}$ \\
\hline EPI696574 & Russia & A/Saint-Petersburg/RII350/2015 & $30 / 11 / 2015$ & $\begin{array}{c}\text { WHO National Influenza Centre } \\
\text { Russian Federation }\end{array}$ & $\begin{array}{c}\text { WHO National Influenza Centre } \\
\text { Russian Federation }\end{array}$ \\
\hline EPI696486 & Russia & A/Saint-Petersburg/RII01/2016 & $19 / 12 / 2015$ & $\begin{array}{l}\text { WHO National Influenza Centre } \\
\text { Russian Federation }\end{array}$ & $\begin{array}{l}\text { WHO National Influenza Centre } \\
\text { Russian Federation }\end{array}$ \\
\hline EPI696494 & Russia & A/Saint-Petersburg/RIlo2/2016 & $21 / 12 / 2015$ & $\begin{array}{c}\text { WHO National Influenza Centre } \\
\text { Russian Federation }\end{array}$ & $\begin{array}{c}\text { WHO National Influenza Centre } \\
\text { Russian Federation }\end{array}$ \\
\hline EPI696502 & Russia & A/Saint-Petersburg/RIlo3/2016 & $21 / 12 / 2015$ & $\begin{array}{c}\text { WHO National Influenza Centre } \\
\text { Russian Federation }\end{array}$ & $\begin{array}{c}\text { WHO National Influenza Centre } \\
\text { Russian Federation }\end{array}$ \\
\hline EPI696510 & Russia & A/Saint-Petersburg/RIlo4/2016 & $21 / 12 / 2015$ & $\begin{array}{c}\text { WHO National Influenza Centre } \\
\text { Russian Federation }\end{array}$ & $\begin{array}{c}\text { WHO National Influenza Centre } \\
\text { Russian Federation }\end{array}$ \\
\hline EPI696166 & Russia & A/IIV-Moscow/155/2015 & $7 / 12 / 2015$ & $\begin{array}{l}\text { D.I. Ivanovsky Research } \\
\text { Institute of virology MoPH of } \\
\text { RF,Moscow }\end{array}$ & $\begin{array}{l}\text { WHO National Influenza Centre } \\
\text { Russian Federation }\end{array}$ \\
\hline EPI696518 & Russia & A/Saint-Petersburg/RIlo5/2016 & $14 / 12 / 2015$ & $\begin{array}{c}\text { WHO National Influenza Centre } \\
\text { Russian Federation }\end{array}$ & $\begin{array}{c}\text { WHO National Influenza Centre } \\
\text { Russian Federation }\end{array}$ \\
\hline EPI696526 & Russia & A/Saint-Petersburg/RIlo6/2016 & $14 / 12 / 2015$ & $\begin{array}{c}\text { WHO National Influenza Centre } \\
\text { Russian Federation }\end{array}$ & $\begin{array}{c}\text { WHO National Influenza Centre } \\
\text { Russian Federation }\end{array}$ \\
\hline EPI696534 & Russia & A/Saint-Petersburg/RIlo7/2016 & $21 / 12 / 2015$ & $\begin{array}{c}\text { WHO National Influenza Centre } \\
\text { Russian Federation }\end{array}$ & $\begin{array}{c}\text { WHO National Influenza Centre } \\
\text { Russian Federation }\end{array}$ \\
\hline EPI696542 & Russia & A/Saint-Petersburg/RIlo8/2016 & $22 / 12 / 2015$ & $\begin{array}{c}\text { WHO National Influenza Centre } \\
\text { Russian Federation }\end{array}$ & $\begin{array}{l}\text { WHO National Influenza Centre } \\
\text { Russian Federation }\end{array}$ \\
\hline EPI696558 & Russia & A/Saint-Petersburg/RII10/2016 & $23 / 12 / 2015$ & $\begin{array}{c}\text { WHO National Influenza Centre } \\
\text { Russian Federation }\end{array}$ & $\begin{array}{c}\text { WHO National Influenza Centre } \\
\text { Russian Federation }\end{array}$ \\
\hline EPI696566 & Russia & A/Saint-Petersburg/RII11/2016 & $24 / 12 / 2015$ & $\begin{array}{c}\text { WHO National Influenza Centre } \\
\text { Russian Federation }\end{array}$ & $\begin{array}{c}\text { WHO National Influenza Centre } \\
\text { Russian Federation }\end{array}$ \\
\hline EPI696222 & Russia & A/IIV-Moscow/166/2015 & $16 / 12 / 2015$ & $\begin{array}{c}\text { D.I. Ivanovsky Research } \\
\text { Institute of virology MoPH of } \\
\text { RF,Moscow }\end{array}$ & $\begin{array}{l}\text { WHO National Influenza Centre } \\
\text { Russian Federation }\end{array}$ \\
\hline EPI696238 & Russia & A/IIV-Moscow/168/2015 & $15 / 12 / 2015$ & $\begin{array}{c}\text { D.I. Ivanovsky Research } \\
\text { Institute of virology MoPH of } \\
\text { RF,Moscow }\end{array}$ & $\begin{array}{l}\text { WHO National Influenza Centre } \\
\text { Russian Federation }\end{array}$ \\
\hline
\end{tabular}


TABLE 2E

Details of the A(H1N1)pdm09 sequences retrieved from the Global Initiative on Sharing All Influenza Data (GISAID)'s EpiFlu Database or GenBank, for haemagglutinin-gene-based phylogenetic analysis in this study

\begin{tabular}{|c|c|c|c|c|c|}
\hline ID & Country & Strain name & $\begin{array}{l}\text { Collection } \\
\text { date }\end{array}$ & Originating laboratory & Submitting laboratory \\
\hline EPI696254 & Russia & A/IIV-Moscow/171/2015 & $17 / 12 / 2015$ & $\begin{array}{l}\text { D.I. Ivanovsky Research } \\
\text { Institute of virology MoPH of } \\
\text { RF,Moscow }\end{array}$ & $\begin{array}{l}\text { WHO National Influenza Centre } \\
\text { Russian Federation }\end{array}$ \\
\hline EPI696294 & Russia & A/IIV-Moscow/178/2015 & $17 / 12 / 2015$ & $\begin{array}{l}\text { D.I. Ivanovsky Research } \\
\text { Institute of virology MoPH of } \\
\text { RF,Moscow }\end{array}$ & $\begin{array}{l}\text { WHO National Influenza Centre } \\
\text { Russian Federation }\end{array}$ \\
\hline EPI696318 & Russia & A/IIV-Moscow/182/2015 & $18 / 12 / 2015$ & $\begin{array}{c}\text { D.I. Ivanovsky Research } \\
\text { Institute of virology MoPH of } \\
\text { RF,Moscow }\end{array}$ & $\begin{array}{l}\text { WHO National Influenza Centre } \\
\text { Russian Federation }\end{array}$ \\
\hline EPI696334 & Russia & A/IIV-Moscow/185/2015 & $18 / 12 / 2015$ & $\begin{array}{c}\text { D.I. Ivanovsky Research } \\
\text { Institute of virology MoPH of } \\
\text { RF,Moscow }\end{array}$ & $\begin{array}{l}\text { WHO National Influenza Centre } \\
\text { Russian Federation }\end{array}$ \\
\hline EPI696366 & Russia & A/IIV-Moscow/189/2015 & $19 / 12 / 2015$ & $\begin{array}{c}\text { D.I. Ivanovsky Research } \\
\text { Institute of virology MoPH of } \\
\text { RF,Moscow }\end{array}$ & $\begin{array}{l}\text { WHO National Influenza Centre } \\
\text { Russian Federation }\end{array}$ \\
\hline EPI696374 & Russia & A/IIV-Moscow/190/2015 & $20 / 12 / 2015$ & $\begin{array}{l}\text { D.I. Ivanovsky Research } \\
\text { Institute of virology MoPH of } \\
\text { RF,Moscow }\end{array}$ & $\begin{array}{l}\text { WHO National Influenza Centre } \\
\text { Russian Federation }\end{array}$ \\
\hline EPI696398 & Russia & A/IIV-Moscow/193/2015 & $21 / 12 / 2015$ & $\begin{array}{l}\text { D.I. Ivanovsky Research } \\
\text { Institute of virology MoPH of } \\
\text { RF,Moscow }\end{array}$ & $\begin{array}{l}\text { WHO National Influenza Centre } \\
\text { Russian Federation }\end{array}$ \\
\hline EPI696406 & Russia & A/IIV-Moscow/194/2015 & $20 / 12 / 2015$ & $\begin{array}{l}\text { D.I. Ivanovsky Research } \\
\text { Institute of virology MoPH of } \\
\text { RF,Moscow }\end{array}$ & $\begin{array}{l}\text { WHO National Influenza Centre } \\
\text { Russian Federation }\end{array}$ \\
\hline EPI696422 & Russia & A/IIV-Moscow/196/2015 & $22 / 12 / 2015$ & $\begin{array}{l}\text { D.I. Ivanovsky Research } \\
\text { Institute of virology MoPH of } \\
\text { RF,Moscow }\end{array}$ & $\begin{array}{l}\text { WHO National Influenza Centre } \\
\text { Russian Federation }\end{array}$ \\
\hline EPI696430 & Russia & A/IIV-Moscow/199/2015 & $22 / 12 / 2015$ & $\begin{array}{l}\text { D.I. Ivanovsky Research } \\
\text { Institute of virology MoPH of } \\
\text { RF,Moscow }\end{array}$ & $\begin{array}{l}\text { WHO National Influenza Centre } \\
\text { Russian Federation }\end{array}$ \\
\hline EPI696446 & Russia & A/IIV-Moscow/203/2015 & $22 / 12 / 2015$ & $\begin{array}{l}\text { D.I. Ivanovsky Research } \\
\text { Institute of virology MoPH of } \\
\text { RF,Moscow }\end{array}$ & $\begin{array}{l}\text { WHO National Influenza Centre } \\
\text { Russian Federation }\end{array}$ \\
\hline EPI696454 & Russia & A/IIV-Moscow/204/2015 & $22 / 12 / 2015$ & $\begin{array}{l}\text { D.I. Ivanovsky Research } \\
\text { Institute of virology MoPH of } \\
\text { RF,Moscow }\end{array}$ & $\begin{array}{l}\text { WHO National Influenza Centre } \\
\text { Russian Federation }\end{array}$ \\
\hline EPI696462 & Russia & A/IIV-Moscow/208/2015 & $23 / 12 / 2015$ & $\begin{array}{l}\text { D.I. Ivanovsky Research } \\
\text { Institute of virology MoPH of } \\
\text { RF,Moscow }\end{array}$ & $\begin{array}{l}\text { WHO National Influenza Centre } \\
\text { Russian Federation }\end{array}$ \\
\hline EPI686526 & Spain & $\mathrm{A} /$ Madrid/1858/2015 & $22 / 12 / 2015$ & $\begin{array}{l}\text { Servicio de Microbiología } \\
\text { Hospital Ramón y Cajal }\end{array}$ & Instituto de Salud Carlos III \\
\hline EPI690296 & Spain & $\mathrm{A} /$ Madrid/1859/2015 & $23 / 12 / 2015$ & $\begin{array}{l}\text { Servicio de Microbiología } \\
\text { Hospital Ramón y Cajal }\end{array}$ & Instituto de Salud Carlos III \\
\hline EPI672780 & Spain & A/Madrid/S013656/2015 & $21 / 10 / 2015$ & Instituto de Salud Carlos III & Instituto de Salud Carlos III \\
\hline EPI674599 & Spain & $\mathrm{A} /$ Madrid/S013670/2015 & $20 / 10 / 2015$ & Instituto de Salud Carlos III & Instituto de Salud Carlos III \\
\hline EPI680490 & Spain & A/Madrid/S013763/2015 & $8 / 12 / 2015$ & Instituto de Salud Carlos III & Instituto de Salud Carlos III \\
\hline EPI699957 & Spain & A/Madrid/41/2016 & $13 / 1 / 2016$ & Instituto de Salud Carlos III & Instituto de Salud Carlos III \\
\hline EPI699959 & Spain & A/Madrid/68/2016 & $12 / 1 / 2016$ & $\begin{array}{l}\text { Servicio de Microbiología } \\
\text { Hospital Ramón y Cajal }\end{array}$ & Instituto de Salud Carlos III \\
\hline EPI699960 & Spain & $\mathrm{A} /$ Madrid/69/2016 & $12 / 1 / 2016$ & $\begin{array}{l}\text { Servicio de Microbiología } \\
\text { Hospital Ramón y Cajal }\end{array}$ & Instituto de Salud Carlos III \\
\hline EPI690298 & Spain & A/Navarra/16004/2015 & $27 / 12 / 2015$ & $\begin{array}{c}\text { Servicio de Microbiología } \\
\text { Complejo Hospitalario de } \\
\text { Navarra }\end{array}$ & Instituto de Salud Carlos III \\
\hline EPI686527 & Spain & A/Navarra/1829/2015 & $15 / 12 / 2015$ & $\begin{array}{c}\text { Servicio de Microbiología } \\
\text { Complejo Hospitalario de } \\
\text { Navarra }\end{array}$ & Instituto de Salud Carlos III \\
\hline EPI686528 & Spain & $\mathrm{A} /$ Navarra/1850/2015 & $17 / 12 / 2015$ & $\begin{array}{c}\text { Servicio de Microbiología } \\
\text { Complejo Hospitalario de } \\
\text { Navarra }\end{array}$ & Instituto de Salud Carlos III \\
\hline
\end{tabular}


TABLE 2F

Details of the A(H1N1)pdm09 sequences retrieved from the Global Initiative on Sharing All Influenza Data (GISAID)'s EpiFlu Database or GenBank, for haemagglutinin-gene-based phylogenetic analysis in this study

\begin{tabular}{|c|c|c|c|c|c|}
\hline ID & Country & Strain name & $\begin{array}{l}\text { Collection } \\
\text { date }\end{array}$ & Originating laboratory & Submitting laboratory \\
\hline EPI690302 & Spain & A/Navarra/26/2016 & $3 / 1 / 2016$ & $\begin{array}{l}\text { Servicio de Microbiología } \\
\text { Complejo Hospitalario de } \\
\text { Navarra }\end{array}$ & Instituto de Salud Carlos III \\
\hline EPI699967 & Spain & A/Navarra/50/2016 & $11 / 1 / 2016$ & $\begin{array}{c}\text { Servicio de Microbiología } \\
\text { Complejo Hospitalario de } \\
\text { Navarra }\end{array}$ & Instituto de Salud Carlos III \\
\hline EPI699973 & Spain & A/Navarra/74/2016 & $14 / 1 / 2016$ & $\begin{array}{c}\text { Servicio de Microbiología } \\
\text { Complejo Hospitalario de } \\
\text { Navarra }\end{array}$ & Instituto de Salud Carlos III \\
\hline EPI699974 & Spain & A/Navarra/75/2016 & $12 / 1 / 2016$ & $\begin{array}{l}\text { Servicio de Microbiología } \\
\text { Complejo Hospitalario de } \\
\text { Navarra }\end{array}$ & Instituto de Salud Carlos III \\
\hline EPI699975 & Spain & A/Navarra/76/2016 & $14 / 1 / 2016$ & $\begin{array}{c}\text { Servicio de Microbiología } \\
\text { Complejo Hospitalario de } \\
\text { Navarra }\end{array}$ & Instituto de Salud Carlos III \\
\hline EPI699977 & Spain & A/Navarra/78/2016 & $14 / 1 / 2016$ & $\begin{array}{c}\text { Servicio de Microbiología } \\
\text { Complejo Hospitalario de } \\
\text { Navarra }\end{array}$ & Instituto de Salud Carlos III \\
\hline EPI672781 & Spain & A/PaisVasco/1683/2015 & $21 / 10 / 2015$ & $\begin{array}{l}\text { Servicio de Microbiología } \\
\text { Hospital Donostia }\end{array}$ & Instituto de Salud Carlos III \\
\hline EPI686529 & Spain & A/PaisVasco/1844/2015 & $15 / 12 / 2015$ & $\begin{array}{l}\text { Servicio de Microbiología } \\
\text { Hospital Donostia }\end{array}$ & Instituto de Salud Carlos III \\
\hline EPI687827 & Slovenia & A/Slovenia/2903/2015 & $26 / 10 / 2015$ & $\begin{array}{l}\text { Laboratory for Virology, } \\
\text { National Institute of Public } \\
\text { Health }\end{array}$ & $\begin{array}{l}\text { Crick Worldwide Influenza } \\
\text { Centre }\end{array}$ \\
\hline $\mathrm{KU}_{558983}$ & $\begin{array}{c}\text { Czech } \\
\text { Republic }\end{array}$ & A/Czech Republic/95/2015 & $1 / 12 / 2015$ & $\begin{array}{l}\text { National Institute of Public } \\
\text { Health }\end{array}$ & $\begin{array}{c}\text { National Institute of Public } \\
\text { Health }\end{array}$ \\
\hline EPI699832 & Greece & A/Athens.GR/2395/2015 & $23 / 12 / 2015$ & Hellenic Pasteur Institute & Hellenic Pasteur Institute \\
\hline EPI699830 & Greece & A/Athens.GR/2407/2015 & $28 / 12 / 2015$ & Hellenic Pasteur Institute & Hellenic Pasteur Institute \\
\hline EPI699827 & Greece & A/Athens.GR/2413/2015 & $29 / 12 / 2015$ & Hellenic Pasteur Institute & Hellenic Pasteur Institute \\
\hline EPI699824 & Greece & A/Athens.GR/12/2016 & $5 / 1 / 2016$ & Hellenic Pasteur Institute & Hellenic Pasteur Institute \\
\hline EPI698911 & Romania & A/Dambovita/190170/2016 & $18 / 1 / 2016$ & Cantacuzino Institute & Cantacuzino Institute \\
\hline EPI698910 & Romania & A/Galati/190006/2016 & $8 / 1 / 2016$ & Cantacuzino Institute & Cantacuzino Institute \\
\hline EPI699021 & Romania & A/Vrancea/190182/2016 & $18 / 1 / 2016$ & Cantacuzino Institute & Cantacuzino Institute \\
\hline EPI699023 & Romania & A/Bucuresti/190324/2016 & $19 / 1 / 2016$ & Cantacuzino Institute & Cantacuzino Institute \\
\hline EPI699059 & Romania & A/Bucuresti/190434/2016 & $23 / 1 / 2016$ & Cantacuzino Institute & Cantacuzino Institute \\
\hline EPI698912 & Romania & A/Dambovita/190171/2016 & $18 / 1 / 2016$ & Cantacuzino Institute & Cantacuzino Institute \\
\hline EPI699024 & Romania & A/Dambovita/190341/2016 & $21 / 1 / 2016$ & Cantacuzino Institute & Cantacuzino Institute \\
\hline EPI699000 & Romania & A/Vrancea/190181/2016 & $11 / 1 / 2016$ & Cantacuzino Institute & Cantacuzino Institute \\
\hline EPI672779 & Spain & A/Aragon/1615/2015 & 29/9/2015 & $\begin{array}{l}\text { Servicio de Microbiología } \\
\text { Hospital Miguel Servet }\end{array}$ & Instituto de Salud Carlos III \\
\hline EPI690293 & Spain & A/Asturias/1862/2015 & $17 / 12 / 2015$ & $\begin{array}{c}\text { Servicio de Microbiología } \\
\text { Hospital Central Universitario } \\
\text { de Asturias }\end{array}$ & Instituto de Salud Carlos III \\
\hline EPI699955 & Spain & A/Baleares/16036/2015 & $30 / 12 / 2015$ & $\begin{array}{c}\text { Servicio de Microbiología } \\
\text { Hospital Universitario Son } \\
\text { Espases }\end{array}$ & Instituto de Salud Carlos III \\
\hline EPI699956 & Spain & A/Baleares/35/2016 & $5 / 1 / 2016$ & $\begin{array}{l}\text { Servicio de Microbiología } \\
\text { Hospital Universitario Son } \\
\text { Espases }\end{array}$ & Instituto de Salud Carlos III \\
\hline EPI690295 & Spain & A/CastillaLaMancha/16013/2015 & $30 / 12 / 2015$ & Instituto de Salud Carlos III & Instituto de Salud Carlos III \\
\hline EPI624748 & Russia & A/St-Petersburg/122/2015 & $26 / 2 / 2015$ & $\begin{array}{c}\text { WHO National Influenza Centre } \\
\text { Russian Federation }\end{array}$ & $\begin{array}{l}\text { Crick Worldwide Influenza } \\
\text { Centre }\end{array}$ \\
\hline EPI624673 & Cameroon & A/Cameroon/15V-3814/2015 & $7 / 5 / 2015$ & Centre Pasteur du Cameroun & $\begin{array}{c}\text { Crick Worldwide Influenza } \\
\text { Centre }\end{array}$ \\
\hline EPI624730 & Norway & A/Norway/1690/2015 & $17 / 3 / 2015$ & WHO National Influenza Centre & $\begin{array}{l}\text { Crick Worldwide Influenza } \\
\text { Centre }\end{array}$ \\
\hline
\end{tabular}

ID: identity; SAR: Special Administrative Region; WHO: World Health Organization. 


\section{TABLE 2G}

Details of the A(H1N1)pdm09 sequences retrieved from the Global Initiative on Sharing All Influenza Data (GISAID)'s EpiFlu Database or GenBank, for haemagglutinin-gene-based phylogenetic analysis in this study

\begin{tabular}{|c|c|c|c|c|c|}
\hline ID & Country & Strain name & $\begin{array}{l}\text { Collection } \\
\text { date }\end{array}$ & Originating laboratory & Submitting laboratory \\
\hline EPI630638 & Mauritius & A/Mauritius/I-463/2015 & $18 / 5 / 2015$ & Central Health Laboratory & $\begin{array}{c}\text { Crick Worldwide Influenza } \\
\text { Centre }\end{array}$ \\
\hline EPI621835 & Madagascar & A/Madagascar/1566/2015 & $15 / 4 / 2015$ & Institut Pasteur de Madagascar & $\begin{array}{c}\text { Crick Worldwide Influenza } \\
\text { Centre }\end{array}$ \\
\hline EPI630634 & $\begin{array}{l}\text { Hong Kong } \\
\text { SAR }\end{array}$ & A/Hong Kong/12243/2015 & $14 / 6 / 2015$ & Government Virus Unit & $\begin{array}{c}\text { Crick Worldwide Influenza } \\
\text { Centre }\end{array}$ \\
\hline EPI630684 & South Africa & A/South Africa/R3723/2015 & $29 / 6 / 2015$ & $\begin{array}{c}\text { Sandringham, National Institute } \\
\text { for Communicable D }\end{array}$ & $\begin{array}{c}\text { Crick Worldwide Influenza } \\
\text { Centre }\end{array}$ \\
\hline EPI630676 & South Africa & A/South Africa/R2977/2015 & $5 / 6 / 2015$ & $\begin{array}{l}\text { Sandringham, National Institute } \\
\text { for Communicable D }\end{array}$ & $\begin{array}{c}\text { Crick Worldwide Influenza } \\
\text { Centre }\end{array}$ \\
\hline EPI630652 & Slovenia & A/Slovenia/1314/15 & $5 / 3 / 2015$ & $\begin{array}{c}\text { Laboratory for Virology, } \\
\text { National Institute of Public } \\
\text { Health }\end{array}$ & $\begin{array}{l}\text { Crick Worldwide Influenza } \\
\text { Centre }\end{array}$ \\
\hline EPI624704 & Russia & A/IIV-Moscow/93/2015 & $10 / 3 / 2015$ & $\begin{array}{c}\text { Ivanovsky Research Institute of } \\
\text { Virology RAMS }\end{array}$ & $\begin{array}{c}\text { Crick Worldwide Influenza } \\
\text { Centre }\end{array}$ \\
\hline $\mathrm{EPI} 589565$ & Jordan & A/Jordan/20241/2015 & $22 / 3 / 2015$ & Laboratory Directorate & $\begin{array}{c}\text { Crick Worldwide Influenza } \\
\text { Centre }\end{array}$ \\
\hline $\mathrm{EPI} 253705$ & Germany & A/Bayern/69/2009 & $1 / 1 / 2009$ & Robert-Koch-Institute & Robert-Koch-Institute \\
\hline $\mathrm{EPI} 278607$ & $\begin{array}{c}\text { New } \\
\text { Zealand }\end{array}$ & A/Christchurch/16/2010 & $12 / 7 / 2010$ & Canterbury Health Services & $\begin{array}{l}\text { WHO Collaborating Centre for } \\
\text { Reference and Research on } \\
\text { Influenza }\end{array}$ \\
\hline $\mathrm{EPI} 319590$ & Russia & A/Astrakhan/1/2011 & $28 / 2 / 2011$ & $\begin{array}{l}\text { WHO National Influenza Centre } \\
\text { Russian Federation }\end{array}$ & $\begin{array}{l}\text { National Institute for Medical } \\
\text { Research }\end{array}$ \\
\hline $\mathrm{EPI}_{319527}$ & Russia & A/St. Petersburg/27/2011 & $14 / 2 / 2011$ & $\begin{array}{l}\text { WHO National Influenza Centre } \\
\text { Russian Federation }\end{array}$ & $\begin{array}{l}\text { National Institute for Medical } \\
\text { Research }\end{array}$ \\
\hline EPI416411 & Norway & A/Norway/120/2013 & $2 / 1 / 2013$ & WHO National Influenza Centre & $\begin{array}{l}\text { National Institute for Medical } \\
\text { Research }\end{array}$ \\
\hline $\mathrm{EPI} 390473$ & $\begin{array}{l}\text { Hong Kong } \\
\text { SAR }\end{array}$ & A/Hong Kong/5659/2012 & $21 / 5 / 2012$ & Government Virus Unit & $\begin{array}{l}\text { National Institute for Medical } \\
\text { Research }\end{array}$ \\
\hline $\mathrm{EPI} 326206$ & $\begin{array}{l}\text { Hong Kong } \\
\text { SAR }\end{array}$ & A/Hong Kong/3934/2011 & $29 / 3 / 2011$ & Government Virus Unit & $\begin{array}{c}\text { National Institute for Medical } \\
\text { Research }\end{array}$ \\
\hline $\mathrm{EPI} 466626$ & South Africa & A/South Africa/3626/2013 & $6 / 6 / 2013$ & $\begin{array}{l}\text { Sandringham, National Institute } \\
\text { for Communicable D }\end{array}$ & $\begin{array}{c}\text { National Institute for Medical } \\
\text { Research }\end{array}$ \\
\hline EPI539472 & Senegal & A/Dakar/04/2014 & $3 / 2 / 2014$ & Institut Pasteur de Dakar & $\begin{array}{l}\text { National Institute for Medical } \\
\text { Research }\end{array}$ \\
\hline $\mathrm{EPI} 417122$ & Senegal & A/Dakar/20/2012 & $9 / 12 / 2012$ & Institut Pasteur de Dakar & $\begin{array}{l}\text { National Institute for Medical } \\
\text { Research }\end{array}$ \\
\hline EPI319447 & $\begin{array}{l}\text { Czech } \\
\text { Republic }\end{array}$ & A/Czech Republic/32/2011 & $18 / 1 / 2011$ & $\begin{array}{c}\text { National Institute of Public } \\
\text { Health }\end{array}$ & $\begin{array}{l}\text { National Institute for Medical } \\
\text { Research }\end{array}$ \\
\hline $\mathrm{EPI} 215957$ & Ukraine & A/Lviv/N6/2009 & $27 / 10 / 2009$ & Ministry of Health of Ukraine & $\begin{array}{l}\text { National Institute for Medical } \\
\text { Research }\end{array}$ \\
\hline $\mathrm{EPI} 320141$ & Russia & A/St. Petersburg/100/2011 & $14 / 3 / 2011$ & $\begin{array}{c}\text { Russian Academy of Medical } \\
\text { Sciences }\end{array}$ & $\begin{array}{c}\text { Centers for Disease Control and } \\
\text { Prevention }\end{array}$ \\
\hline EPI626148 & Bangladesh & A/Bangladesh/3003/2015 & $4 / 5 / 2015$ & $\begin{array}{c}\text { Institute of Epidemiology } \\
\text { Disease Control and Research } \\
\text { (IEDCR) and Bangladesh } \\
\text { National Influenza Centre (NIC) }\end{array}$ & $\begin{array}{c}\text { Centers for Disease Control and } \\
\text { Prevention }\end{array}$ \\
\hline EPI626140 & Bangladesh & A/Bangladesh/01/2015 & $10 / 5 / 2015$ & $\begin{array}{l}\text { Institute of Epidemiology } \\
\text { Disease Control and Research } \\
\text { (IEDCR) and Bangladesh } \\
\text { National Influenza Centre (NIC) }\end{array}$ & $\begin{array}{c}\text { Centers for Disease Control and } \\
\text { Prevention }\end{array}$ \\
\hline $\mathrm{EPI} 176620$ & $\begin{array}{l}\text { United } \\
\text { States }\end{array}$ & A/California/07/2009 & $9 / 4 / 2009$ & Naval Health Research Center & $\begin{array}{c}\text { Centers for Disease Control and } \\
\text { Prevention }\end{array}$ \\
\hline EPI624468 & $\begin{array}{l}\text { French } \\
\text { Guiana }\end{array}$ & A/Guyane/1759/2015 & $9 / 4 / 2015$ & Institut Pasteur & Institut Pasteur \\
\hline
\end{tabular}


The data supporting the predominance of the $6 \mathrm{~B} .1$ subclade stem from the subset of 12 European countries that reported virus characterisation data referring to sequences available in publically accessible databases. These countries are well spread across Europe which corroborates the conclusion of widespread 6B.1 subclade circulation. Data from the WHO Collaborating Centres indicate that the new subgroup remains antigenically similar to the vaccine component $A$ / California/7/2009 [1], but some recent $A\left(\mathrm{H}_{1} \mathrm{~N}_{1}\right)$ pdmog viruses within the 6B.1 and 6B.2 subclades reacted poorly with sera from individuals vaccinated with $A$ / California/7/2009-like-strain-containing vaccine [15].

The emergence of a new $\mathrm{A}\left(\mathrm{H}_{1} \mathrm{~N}_{1}\right)$ pdmog subclade may eventually affect the susceptibility of the population to the currently circulating $\mathrm{A}\left(\mathrm{H}_{1} \mathrm{~N}_{1}\right)$ pdmog viruses, e.g. by viruses drifting closer to become immune escape variants. It is not clear whether the emergence and predominance of subclade 6B.1 has been driven by immune selection or what its impact on vaccine effectiveness may be and this needs assessment e.g. by generating lineage-specific estimation of vaccine effectiveness. Early vaccine effectiveness estimates for $A\left(\mathrm{H}_{1} \mathrm{~N}_{1}\right)$ pdmog this season compared with the previous ones are not significantly different [16] from previous seasons. As to the severity observed this season [1-4], similar observations have been made also in earlier seasons e.g. in 2010/11 in the United Kingdom, which experienced notably severe $A\left(\mathrm{H}_{1} \mathrm{~N}_{1}\right)$ pdmog impact in the first post-pandemic season.

Notably, recent studies have demonstrated that antigenic change in $\mathrm{A}\left(\mathrm{H}_{1} \mathrm{~N}_{1}\right)$ pdmog viruses is mainly caused by single amino acid substitutions affecting the loop located adjacent to the receptor binding site [17]; eight of the 215 analysed 2015/16 viruses possessed such substitutions, all six of the viruses in subclade 6B.2 and two in 6B subgroup, that do not belong to any of the newly emerged subclades.

Further enhancement of the antigenicity and virulence of influenza virus has been attributed to shielding of the major antigenic epitopes by alteration of $\mathrm{N}$-linked glycosylation sites [18]. D127E substitution seen in 6B.2 has been associated with antigenic change of other influenza viruses through modelling [17]. The change at position 173 ( $\mathrm{V}_{173}$ ) also in the 6B.2 subclade of viruses is located in antigenic site Ca1 (position 169-173), and therefore a change here could contribute to antigen drift. It has been proposed that the evolution of $A\left(\mathrm{H}_{1} \mathrm{~N}_{1}\right)$ pdmog will involve the acquisition of additional glycosylations, as for former seasonal $\mathrm{A}\left(\mathrm{H}_{1} \mathrm{~N}_{1}\right) \mathrm{HA}$ [19]. Noteworthy, $80 \%$ of the analysed $\mathrm{HA}$ sequences have gained a potential glycosylation site $\mathrm{S} 162 \mathrm{~N}$. No D222G/E/N substitutions were detected, nor N129D which was recently identified in India in two severe or fatal cases [9]. If the emerging groups continue to diversify from the vaccine component, their antigenic properties may change and the vaccine effectiveness might be reduced. WHO recommended not to change the vaccine component of $A\left(\mathrm{H}_{1} \mathrm{~N}_{1}\right)$ pdmog for the northern hemisphere $2016 / 17$ season [20].

Early vaccine effectiveness estimates for 2015/16 are not yet available for $\mathrm{A}\left(\mathrm{H}_{3} \mathrm{~N}_{2}\right)$ and $\mathrm{B}$ viruses which have been detected in lower numbers in most countries. The $B /$ Victoria virus component is available only in the quadrivalent vaccines in the northern hemisphere for this season. As the majority of the countries use trivalent vaccines, the lineage switch from B/Yamagata to $B /$ Victoria may contribute to lower vaccine effectiveness against influenza $B$. For $A\left(\mathrm{H}_{3} \mathrm{~N}_{2}\right)$, the current component of influenza vaccines is expected to have improved vaccine effectiveness compared with the two previous seasons $[21,22]$. In the southern hemisphere, seasonal influenza vaccine has been demonstrated to have an overall effectiveness against $\mathrm{A}\left(\mathrm{H}_{3} \mathrm{~N}_{2}\right)$ of $36 \%$ (95\% confidence interval $(\mathrm{Cl}): 11-54)$ ) for general practice encounters and 50\% (95\%-Cl: $20-68)$ for hospitalisations in 2015 [23]. Despite the changes in the genetic makeup of influenza $A\left(\mathrm{H}_{1} \mathrm{~N}_{1}\right)$ pdmog viruses and the predominance of $\mathrm{B} /$ Victoria lineage over $\mathrm{B} /$ Yamagata lineage, seasonal influenza vaccine remains the single most effective measure to prevent severe outcomes of influenza.

\section{Disclaimer}

The authors alone are responsible for the views expressed in this article and they do not necessarily represent the views, decisions or policies of the institutions with which they are affiliated.

Members of the World Health Organization European Region and European Influenza Surveillance Network of the reporting countries

Belgium: Isabelle Thomas, Anneleen Hombrouck, Nathalie Bossuyt, Sarah Moreels, Viviane Van Casteren; Croatia: Vladimir Drazenovic; Czech Republic: Martina Havlickova, Helena Jiřincová, Jan Kyncl; Denmark: Ramona Trebbien, Lisbet Krause Knudsen, Tyra Grove Krause, Thea Kølsen Fischer; Finland: Niina Ikonen, Anu Haveri, Outi Lyytikäinen, Satu Murtopuro; Germany: Brunhilde Schweiger, Marianne Wedde, Barbara Biere, Silke Buda; Greece: Athanasios Kossyvakis, Spala Georgia, Andreas Mentis, Nikolaos Malisiovas; Ireland: Lisa Domegan, Joan O’Donnell, Darina O־Flanagan, Joanne Moran, Grainne Tuite, Margaret Duffy, Jeff Connell, Cillian De Gascun; Latvia: Raina Nikiforova, Gatis Pakarna, Natalija Zamjatina; Netherlands: Adam Meijer, Anne Teirlinck, Frederika Dijkstra, Ge Donker, Guus Rimmelzwaan, Marit de Lange; Norway: Olav Hungnes, Karoline Bragstad, Siri Helene Hauge, Ragnhild Tønnessen, Susanne Gjeruldsen Dudman, Karine Nordstrand, Tora Schraffenberg, Gry Grøneng.; Portugal: Raquel Guiomar, Pedro Pechirra, Paula Cristovão, Inês Costa, Patrícia Conde, Baltazar Nunes, Ana Rodrigues; Romania: Emilia Lupulescu, Rodica Popescu, Odette Popovici, Florin Popovici; Russia: Elena Burtseva, Anna Sominina, Andrey Komissarov, Artem Fadeev, Elena Kirillova; Slovakia: Elena Tichá, Edita Staroňová, Ján Mikas; Slovenia: Katarina Prosenc, Nataša Berginc, Maja Sočan; Spain: Inmaculada Casas, Amparo Larrauri, Francisco Pozo, Concha Delgado, Jesús Oliva; Sweden: Mia Brytting, Annasara Carnahan, Åsa Wiman; Switzerland: Rita Born, Samuel Cordey; United Kingdom: Peter Coyle, Alasdair MacLean, Rory Gunson, Mary Sinnanthamby, Praveen Sebastian Pillai, Cathriona Kearns, Maria Zambon, Christopher Nugent, Catherine Moore, Nick 
Phin, Richard Pebody, Simon de Lusignan, Simon Cottrell, Jim McMenamin, Lucy Jessop; European Centre for Disease Prevention and Control: Cornelia Adlhoch, Adrian Prodan, René Snacken, Pasi Penttinen; WHO Collaborating Centre for Reference and Research on Influenza London: John McCauley, Rod Daniels; WHO Regional Office for Europe: Dmitriy Pereyaslov, Sergejs Nikišins.

\section{Acknowledgements}

We acknowledge the authors, originating and submitting laboratories of the sequences from GISAID's EpiFlu Database on which the phylogenetic analysis is based (Figure 1; Accession numbers shown in Table 2). All submitters of data may be contacted directly via the GISAID website www.gisaid.org.

We thank Julien Beauté, Phillip Zucs, Denis Coulombier and Piotr Kramarz (ECDC) for improving this article, and Torstein Aune (Norway) for technical assistance in the analysis. We would also like to thank Caroline Brown from the WHO Regional Office for Europe for our joint collaboration on influenza surveillance.

We would additionally like to acknowledge all members of the Spanish Influenza Surveillance System (SISS) for the contribution in this study. Sequences from Spain were obtained in the Centro Nacional de Microbiología (ISCIII) from influenza viruses sent by the following laboratories: Hospital Miguel Servet de Zaragoza-Aragón, Hospital Universitario Central de Asturias, Hospital Universitario Son Espases de Palma de Mallorca-Baleares, Hospital San Pedro de Alcántara CáceresExtremadura, Hospital Donostia-País Vasco, Hospital San Pedro Logroño-La Rioja, Complejo Hospitalario Universitario de Vigo-Galicia.

\section{Conflict of interest}

None declared.

\section{Authors' contributions}

Eeva Broberg: Data extraction, data maintenance, first draft of the manuscript, study design, revisions of the article. Angeliki Melidou: data processing, phylogenetic analysis and text. Katarina Prosenc: data processing, analysis, text. Karoline Bragstad: data processing, amino acid analysis, text. Olav Hungnes: data processing, analysis, text. ECDC and WHO Regional Office for Europe staff: influenza surveillance data maintenance, management and analysis. Country experts: surveillance systems, data collection, data analysis at national level and reporting to TESSy.

\section{References}

1. World Health Organization (WHO). Risk Assessment - Seasonal Influenza A(H1N1)pdmo9 2016. Geneva: WHO. [Accessed 22 Feb 2016]. Available from: http://www.who.int/influenza/ publications/riskassessment AH1N1pdmo9 201602/en/

2. The Public Health Agency of Sweden. Ovanligt många allvarligt sjuka i influensa 2016. Swedish. [Accessed 22 Feb 2016]. Available from: http://www.folkhalsomyndigheten. se/nyheter-och-press/nyhetsarkiv/2016/februari/ ovanligt-manga-allvarligt-sjuka-i-influensa/

3. European Centre for Disease Prevention and Control (ECDC). Risk assessment Seasonal influenza 2015-2016 in the EU/ EEA countries 2016. Stockholm: ECDC. [Accessed 26 Feb 2016]. Available from: http://ecdc.europa.eu/en/publications/ Publications/seasonal-influenza-risk-assessment-2015-2016. pdf

4. Centers for Disease Control and Prevention (CDC). CDC health advisory. Flu Season Begins: Severe Influenza Illness Reported CDC urges rapid antiviral treatment of very ill and high risk suspect influenza patients without waiting for testing 2016. Atlanta: CDC. [Accessed 22 Feb 2016]. Available from: http:// emergency.cdc.gov/han/hano0387.asp

5. World Health Organization Regional Office for Europe (WHO/ Europe). Risk assessment of the 2015-2016 influenza season in the WHO European Region, week 40/2015 to week 04/2016 2016. Copenhagen: WHO/Europe. [Accessed 8 Mar 2016]. Available from: http://www.euro.who.int/__data/assets/pdf_ file/o011/301115/Risk-assessment-influenza-season-week4015-to-weeko4-16.pdf

6. WHO European Region and the European Influenza Surveillance Network,Broberg E, Snacken R, Adlhoch C, Beauté J, Galinska $M$, Pereyaslov D, et al. . Start of the $2014 / 15$ influenza season in Europe: drifted influenza $\mathrm{A}\left(\mathrm{H}_{3} \mathrm{~N}_{2}\right)$ viruses circulate as dominant subtype.Euro Surveill. 2015;20(4):21023. DOI: 10.2807/1560-7917.ES2015.20.4.21023 PMID: 25655052

7. European Centre for Disease Prevention and Control (ECDC). Influenza virus characterisation, Summary Europe, September 2015. Stockholm: ECDC; 2015. [Accessed 7 Mar 2016]. Available from: http://ecdc.europa.eu/en/publications/Publications/ influenza-virus-characterisation-september-2015.pdf

8. European Centre for Disease Prevention and Control (ECDC). Influenza virus characterisation, summary Europe, December 2015. Stockholm: ECDC; 2016. [Accessed 22 Feb 2016]. Available from: http://ecdc.europa.eu/en/publications/ Publications/Influenza-Virus-Characterisation-December-2015. pdf

9. Parida M, Dash PK, Kumar JS, Joshi G, Tandel K, Sharma S, et al. Emergence of influenza $\mathrm{A}\left(\mathrm{H}_{1} \mathrm{~N}_{1}\right)$ pdmog genogroup $6 \mathrm{~B}$ and drug resistant virus, India, January to May 2015. Euro Surveill. 2016;21(4):30124. DOI: 10.2807/1560-7917.ES.2016.21.5.30124 PMID: 26876980

10. World Health Organization (WHO). Global epidemiological surveillance standards for influenza 2013. Geneva: WHO. [Accessed 22 Feb 2016]. Available from: http://www.who. int/influenza/resources/documents/WHO_Epidemiological Influenza_Surveillance_Standards_2014.pdf

11. European Centre for Disease Prevention and Control (ECDC). Influenza surveillance in Europe 2010-2011. Stockholm: ECDC; 2011. [Accessed 22 Feb 2016]. Available from: http:// ecdc.europa.eu/en/publications/Publications/111209_SUR_ Influenza surveillance Europe\%20 2010 2012.pdf

12. Snacken R, Broberg E, Beauté J, Lozano JE, Zucs P, Amato-Gauci AJ. Influenza season 2012-2013 in Europe: moderate intensity, mixed (sub)types.Epidemiol Infect. 2014;142(9):1809-12. DOI: 10.1017/So950268814001228 PMID: 24814635

13. European Centre for Disease Prevention and Control (ECDC). Influenza in Europe - Season 2013-2014. Stockholm: ECDC; 2014. [Accessed 22 Feb 2016]. Available from: http://ecdc. europa.eu/en/publications/Publications/Influenza-2013-14season-report.pdf

14. European Centre for Disease Prevention and Control (ECDC). Influenza virus characterisation, December 2013. Stockholm: ECDC; 2014. [Accessed 22 Feb 2016]. Available from: http:// ecdc.europa.eu/en/publications/Publications/influenza-virus characterisation-dec-2013.pdf

15. World Health Organization (WHO). Recommended composition of influenza virus vaccines for use in the 2016-2017 northern hemisphere influenza season 2016. Geneva: WHO. [Accessed 25 Feb 2016]. Available from: http://www.who.int/influenza/ vaccines/virus/recommendations/201602_recommendation. pdf

16. Kissling $E$, Valenciano $M$. Early influenza vaccine effectiveness results 2015-16: I-MOVE multicentre case-control study. Euro Surveill. 2016;21(6):30134. DOI: 10.2807/1560-7917. ES.2016.21.6.30134 PMID: 26898240

17. Koel BF, Mögling R, Chutinimitkul S, Fraaij PL, Burke DF, van der Vliet S, et al. Identification of amino acid substitutions supporting antigenic change of influenza $\mathrm{A}\left(\mathrm{H}_{1} \mathrm{~N}_{1}\right)$ pdmog viruses. J Virol. 2015;89(7):3763-75. DOI: 10.1128/JVI.02962-14 PMID: 25609810

18. Tate MD, Job ER, Deng YM, Gunalan V, Maurer-Stroh S, Reading PC. Playing hide and seek: how glycosylation of the influenza virus hemagglutinin can modulate the immune response to infection.Viruses. 2014;6(3):1294-316. DOI: 10.3390/v6031294 PMID: 24638204

19. Wei CJ, Boyington JC, Dai K, Houser KV, Pearce MB, Kong WP, et al. Cross-neutralization of 1918 and 2009 influenza viruses: role of glycans in viral evolution and vaccine design. Sci Transl Med. 2010;2(24):24ra21. DOI: 10.1126/scitranslmed.3000799 PMID: 20375007

20. World Health Organization (WHO),. Recommended composition of influenza virus vaccines for use in the 2016-2017 northern hemisphere influenza season.Wkly Epidemiol Rec. 2016;91(10):121-32.http://reliefweb.int/sites/reliefweb.int/ files/resources/wer9110.pdfPMID: 26971356 
21. I-MOVE Multicentre Case Control Study Team,Valenciano M,

Kissling E, Reuss A, Jiménez-Jorge S, Horváth JK, Donnell JM, et al. . The European I-MOVE Multicentre 2013-2014 Case-Control Study. Homogeneous moderate influenza vaccine effectiveness against $A\left(\mathrm{H}_{1} \mathrm{~N}_{1}\right)$ pdmog and heterogenous results by country against $\mathrm{A}\left(\mathrm{H}_{3} \mathrm{~N}_{2}\right)$.Vaccine. 2015;33(24):2813-22. DOI: 10.1016/j. vaccine.2015.04.012 PMID: 25936723

22. Joan $0^{`}$ Donell,Valenciano M, Kissling E, Reuss A, Rizzo C, Gherasim A, Horváth JK, et al. . Vaccine effectiveness in preventing laboratory-confirmed influenza in primary care patients in a season of co-circulation of influenza $A\left(\mathrm{H}_{1} \mathrm{~N}_{1}\right)$ pdmo9, B and drifted $\mathrm{A}\left(\mathrm{H}_{3} \mathrm{~N}_{2}\right)$, I-MOVE Multicentre CaseControl Study, Europe 2014/15.Euro Surveill. 2016;21(7):30139. DOI: $10.2807 / 1560-7917 . E S .2016 .21 .7 .30139$ PMID: 26924024

23. SHIVERS, Bissielo A, Pierse N, Huang QS, Thompson MG, Kelly $\mathrm{H}$, Mishin VP, et al. . Effectiveness of seasonal influenza vaccine in preventing influenza primary care visits and hospitalisation in Auckland, New Zealand in 2015: interim estimates.Euro Surveill. 2015;21(1):30101. DOI: 10.2807/15607917.ES.2016.21.1.30101 PMID: 26767540

\section{License and copyright}

This is an open-access article distributed under the terms of the Creative Commons Attribution (CC BY 4.0) Licence. You may share and adapt the material, but must give appropriate credit to the source, provide a link to the licence, and indicate if changes were made.

This article is copyright of the authors, 2016. 